\title{
Analysis of Indole-3-acetic Acid (IAA) Production in Klebsiella by LC-MS/MS and the Salkowski Method
}

Shraddha Gang ${ }^{1,2}$, Sheetal Sharma², Meenu Saraf ${ }^{2, *}$, Martin Buck ${ }^{1}$ and Jörg Schumacher ${ }^{1, *}$

${ }^{1}$ Dept. of Life Science, Faculty of Natural Sciences, Imperial College, London SW7 2AZ, UK; ${ }^{2}$ Dept. of Microbiology and Biotechnology, School of Sciences, Gujarat University, Ahmedabad 380009, India *For correspondence: j.schumacher@ic.ac.uk; sarafmeenu@gmail.com

[Abstract] Many rhizobacteria isolated from plant rhizosphere produce various phytohormones in the form of secondary metabolites, the most common of which is Indole-3-acetic acid (IAA). Here, we detail analytical protocols of IAA detection and quantification, in vitro and in situ, as recently applied to Klebsiella SGM 81, a rhizobacterium isolated from the rhizosphere of Dianthus caryophyllus (a commercially important flower across the globe). Specifically, we describe a detailed protocol for a colorimetric assay using the Salkowski reagent method, which can be used to screen for the presence of Indole compounds. To further detect and quantify IAA, a highly accurate analytical approach of LCMS/MS is used. To detect the presence of IAA around the root system of Dianthus caryophyllus, in situ staining of plant roots is done using Salkowski reagent.

Keywords: Indole-3-acetic acid, Klebsiella, Salkowski reagent, LC-MS/MS, Tryptophan, Spectrophotometer

[Background] The bacterial auxin in the form of Indole-3 Acetic Acid (IAA) is a product of L-tryptophan metabolized by bacteria (Lynch, 1985). The group of bacteria known as plant growth promoting rhizobacteria (PGPR) specifically residing in the vicinity of the roots depend on tryptophan being present in the root exudates of plants (Kravchenko et al., 2004; Kamilova et al., 2006). These PGPR use IAA as a signal to interact with plant roots and to colonize the plant parts. This signaling feature of IAA is thought to effect on the physiology of the bacteria (Spaepen et al., 2007).

Different methods are found in the literature to detect the biosynthesis of IAA. Gordon and Weber (1951) were the first to provide a colorimetric assay using Salkowski reagent for the detection of IAA. This method has since been widely used for detecting IAA from microorganisms. Salkowski reagent is a mixture of $0.5 \mathrm{M}$ ferric chloride $\left(\mathrm{FeCl}_{3}\right)$ and $35 \%$ perchloric acid $\left(\mathrm{HClO}_{4}\right)$ which upon reaction with IAA yields pink color, due to IAA complex formation with and reduction of $\mathrm{Fe}^{3+}$ (Kamnev et al., 2001). The color developed by positive reaction indicates the presence of various indole compounds as a product of tryptophan metabolism. Apart from the colorimetric assay, other methods for IAA estimation from bacteria and plant are High Performance Liquid Chromatography (HPLC) (Perrig et al., 2007), Liquid Chromatography Electrospray Ionization Tandem Mass Spectrometric (LC-ESI-MS/MS) (Chiwocha et al., 2003), and by High Performance Thin Layer Chromatography HPTLC (Goswami et al., 2015). Liquid Chromatography (LC) is the preferred approach to determine the concentration of IAA and to confirm its purity with high accuracy and standardization. LC coupled with various mass spectrometry 
detectors are powerful tools for IAA analysis. Because of the high sensitivity and selectivity, Mass Spectrometry detectors are most commonly coupled with LC. One of the important benefits of LC-MS is that analysis and separation of compounds can be achieved in a continuous manner eliminating the step of purification (Kallenbach et al., 2009).

\section{Materials and Reagents}

1. $100-1,000 \mu l$ pipette tips (Sigma-Aldrich)

2. Test tube racks (Sigma-Aldrich, catalog number: Z334146)

3. Screw-cap tubes and caps (Sigma-Aldrich, catalog number: AXYSCT10MLS)

4. Pyrex test tube $20 \mathrm{ml}$ (Sigma-Aldrich, catalog number: CLS980016)

5. UV quartz cuvette semi-micro square, $1.4 \mathrm{ml}$, PTFE stopper (Sigma-Aldrich, catalog number: Z276707)

6. Sterile syringe filters with a pore size of $0.2 \mu \mathrm{m}$ (Fisher Scientific, catalog number: 720-1230)

7. Centrifuge tube with screw cap, capacity $10 \mathrm{ml}$ (Sigma-Aldrich, catalog number: SIAL301NN10R)

8. Pyrex glass serological pipettes, capacity $5 \mathrm{ml}$ (Sigma-Aldrich, catalog number: Z653829)

9. Amber storage bottles (Sigma-Aldrich, Supelco, catalog number: $23230-U$ )

10. Klebsiella SGM 81 strain (isolated from the rhizosphere of Dianthus caryophyllus)

11. Distilled water

12. Nutrient broth (Microbiology grade) (Sigma-Aldrich, catalog number: 70122-500G), its composition and final $\mathrm{pH}$ see below:

\begin{tabular}{ll}
\hline Grade & For microbiology \\
\hline Composition & $\mathrm{D}(+)$-glucose, $1 \mathrm{~g} / \mathrm{L}$ \\
\hline & Peptone, $15 \mathrm{~g} / \mathrm{L}$ \\
& Sodium chloride, $6 \mathrm{~g} / \mathrm{L}$ \\
\hline & Yeast extract, $3 \mathrm{~g} / \mathrm{L}$ \\
\hline Final $\mathrm{pH}$ & $7.5 \pm 0.2\left(25^{\circ} \mathrm{C}\right)$ \\
\hline
\end{tabular}

13. $\mathrm{FeCl}_{3}$ reagent grade (Sigma-Aldrich, CAS: 7705-08-0)

14. Perchloric acid 70\% (Sigma-Aldrich, CAS: 7601-90-3)

15. Methanol (laboratory grade) (Sigma-Aldrich, CAS: 67-56-1)

16. $\mathrm{HCl}$ (ACS grade) (Sigma-Aldrich, CAS: 7647-01-0)

17. L-tryptophan (traceCERT grade) (Sigma-Aldrich, CAS: 73-22-3)

18. Acetic acid glacial (Sigma-Aldrich, CAS: 64-19-7)

19. Ethyl acetate (grade anhydrous) (Sigma-Aldrich, CAS: 141-78-6)

20. Indole-3-Acetic acid (IAA) (Sigma-Aldrich, CAS: 87-51-4) 
21. Murashige and Skoog medium (Sigma-Aldrich)

22. Acetonitrile

23. Ammonium formate

24. $\mathrm{CH}_{3} \mathrm{CN}$

25. Formic acid

26. Ethanol

27. Agarose

28. Culture media (see Recipes)

29. Salkowski reagent (see Recipes)

\section{Equipment}

1. Spatula (Thermo Fisher, catalog number: F36711-0012)

2. Nichipet Eco pipette, 100-1,000 $\mu$ l volume (Sigma-Aldrich, catalog number: Z710199)

3. Measuring cylinder (Sigma-Aldrich, catalog number: Z324361-2EA)

4. $250 \mathrm{ml}$ conical flask (Sigma-Aldrich, catalog number: Z723088-1EA)

5. Rotary Vacuum Flash Evaporator (Buchhi Type) (Jain Scientific Glass, 1188)

6. Balance

7. Agilent 1100 LC system and an ABSciex 6500 Qtrap MS [Chromatography was on a Phenomenex Luna C18 column (100 $\mathrm{mm} \times 2 \mathrm{~mm} \times 3 \mu \mathrm{m})]$

8. UV-spectrophotometer (Systronics, 166)

9. Microcentrifuge (Thermo scientific, Pico 17)

10. Magnetic stirrer (Remi, $1 \mathrm{MLH}$ )

11. Vortex cyclo mixer (Remi, CM 101)

\section{Software}

1. Analyst 1.6.1 (AB Sciex)

\section{Procedure}

A. IAA screening and quantification from Klebsiella SGM 81 using Salkowski reagent method

1. Culture preparation

a. Take preculture medium (without tryptophan) and inoculate it with a single colony of SGM 81 strain, or a loopful of glycerol stock of the strain.

b. Incubate the inoculated pre-culture medium at $30^{\circ} \mathrm{C}, 120 \mathrm{rpm}$ overnight. This will result in the young culture of the Klebsiella SGM 81 strain which can be used for inoculating IAA production medium (test media).

c. Add $100 \mu \mathrm{l}$ of the young culture in test medium and vortex it to get a uniform suspension. 
d. Incubate it under dark conditions (by wrapping the container with newspaper/aluminum foil) at $30^{\circ} \mathrm{C}$ in shaking condition at $120 \mathrm{rpm}$.

e. Incubation lasts for 24-96 $\mathrm{h}$ in dark condition depending on IAA production ability of the strain. Klebsiella SGM 81 produces IAA till $72 \mathrm{~h}$ and subsequent decrease in the production in recorded after that.

2. Indole quantification using Salkowaski reagent

a. After $24 \mathrm{~h}$, take out $1.5 \mathrm{ml}$ of sample culture broth and transfer to an Eppendorf tube.

b. Centrifuge at $16,278 \times g$ for 5 min using a microcentrifuge.

c. Carefully withdraw $1 \mathrm{ml}$ supernatant and transfer to a new test tube.

d. Mix equal volume $(1 \mathrm{ml})$ of Salkowski reagent, vortex gently and incubate the reaction at $30^{\circ} \mathrm{C}$ in a dark condition for $30 \mathrm{~min}$.

e. In a new test tube replace the supernatant with $1 \mathrm{ml}$ of control uninoculated medium and mix with $1 \mathrm{ml}$ of Salkowski reagent. This serves as blank.

f. The presence of IAA is detected by measuring pink color development after 30 min (some strains may develop red color as shown in Figure 1).

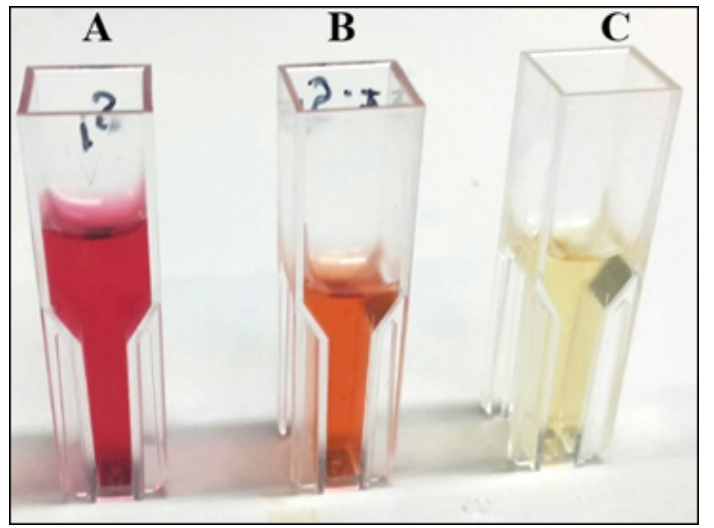

Figure 1. Color development due to Indole-Salkowski reagent reaction. A. Klebsiella SGM

81. B. SGM09. C. Control uninoculated media.

g. Use uninoculated medium to set the blank. Measure the color intensity spectrophotometrically at $536 \mathrm{~nm}$ using cuvette.

h. Compare the optical density of the test sample with a standard IAA curve $\left(10-100 \mu \mathrm{g} \cdot \mathrm{ml}^{-1}\right)$ to calculate the concentration.

i. Repeat the process at similar intervals until the optical density begins to decrease, indicating no further production of IAA.

B. Analysis of IAA by liquid chromatography-mass spectrometry (LC-MS/MS)

1. Sample preparation

a. Prepare $100 \mathrm{ml}$ nutrient broth medium supplemented with $0.5 \mathrm{~g}$ of L-tryptophan in a $250 \mathrm{ml}$ Erlenmeyer flask. 
b. Add $100 \mu \mathrm{l}$ of the young culture in test medium and vortex it to get a uniform suspension.

c. Incubate it under dark conditions (by wrapping the container with newspaper/aluminum foil) at $30^{\circ} \mathrm{C}$ in shaking condition at $120 \mathrm{rpm}$ for $72 \mathrm{~h}$.

d. To separate the cells, centrifuge at $4{ }^{\circ} \mathrm{C}$ for $20 \mathrm{~min}$ at $2,800 \times \mathrm{g}$ and collect $1,000 \mathrm{ml}$ supernatant.

e. Transfer the supernatant to a $250 \mathrm{ml}$ screw-cap glass bottle. Acidify it by adding 2-3 drops of $1 \mathrm{~N}$ concentrated $\mathrm{HCl}$ to reach $\mathrm{pH}$ 2.5-3.

f. To extract IAA, add double the volume of ethyl acetate to the acidified supernatant and shake vigorously for $5 \mathrm{~min}$ (alternatively, a separating funnel can be used). Let the mixture stand at room temperature for $10 \mathrm{~min}$ to get the top layer of ethyl acetate. Use this layer for further treatment.

g. In a rotary evaporator, set water bath temperature to boiling temperature of ethyl acetate i.e., $77.1^{\circ} \mathrm{C}$ (alternatively, ethyl acetate phase can be vacuum dried in a rotational evaporator at $40{ }^{\circ} \mathrm{C}$ ). The time for drying of the solvent system varies according to the sample volume.

h. Transfer the ethyl acetate layer to the round bottom flask and adjust its rotation to avoid any bumping of the liquid sample. "Bumping is the phenomenon in chemistry where homogenous liquids boiled in a test tube or other container will super heat and, upon nucleation rapid boiling will expel the liquid from the container (Wikipedia)"-This would leave the IAA escaping the flask into the condenser tube attached with the round bottom flask.

i. Upon complete evaporation of the liquid ethyl acetate, pure IAA is left behind in the crystalline form attached to the bottom of the rotary flask.

j. Switch off all the units of the rotary evaporator and remove the round bottom flask. Redissolve the crystalline IAA in $5 \mathrm{ml}$ of $20 \%$ methanol and store at $-20^{\circ} \mathrm{C}$ for future use.

2. LC-MS/MS analysis

a. Filter the stored methanol extract $(100 \mu \mathrm{l})$ using sterile syringe filters of $0.2 \mu \mathrm{m}$ so as to separate insoluble particles and larger compounds if any. This is the final analysis sample.

b. Phenomenex Luna C18(2) column (100 $\mathrm{mm} \times 2 \mathrm{~mm} \times 3 \mu \mathrm{m})$ at temperature of $50^{\circ} \mathrm{C}$ is used as chromatography system.

c. A gradient solvent system of $10 \%$ solvent $A$ ( $2 \%$ acetonitrile, $10 \mathrm{mM}$ ammonium formate, $\mathrm{pH} 4.2)$ to $90 \%$ solvent $\mathrm{B}\left(94.9 \% \mathrm{CH}_{3} \mathrm{CN}, 5 \% \mathrm{H}_{2} \mathrm{O}, 0.1 \%\right.$ formic acid) is used for the column over $10 \mathrm{~min}$ at a flow rate of $250 \mathrm{ml} / \mathrm{min}$.

d. Wash the column with $90 \%$ solvent B for 3 min and then re-equilibrate with $90 \%$ solvent $A$ for 6 min.

e. Use $10 \mu \mathrm{l}$ injections to load the sample for analysis.

f. The MS is configured with a Turbo Spray Ion Drive source where the source temperature is set to $500{ }^{\circ} \mathrm{C}$ and the ion spray voltage to $5,500 \mathrm{~V}$.

g. Analyze IAA by Multiple Reaction Monitoring (MRM) in positive mode using a transition of $176>130$ with collision energy of $20 \mathrm{eV}$. Transition of $176>130$ means the specific pairs 
of mass to charge $(\mathrm{m} / \mathrm{z})$ values associated to the precursor and fragment ions. In our case the Q1 is set to a value of $176 \mathrm{amu}$ and the Q3 to $130 \mathrm{amu}$, the collision energy was $20 \mathrm{mV}$.

$\mathrm{h}$. Set the declustering potential, exit potential and collision cell exit potential at $30 \mathrm{~V}, 10 \mathrm{~V}$ and $10 \mathrm{~V}$ respectively.

C. In Situ Salkowski staining

1. Prepare Murashige and Skoog (MSM) basal salt medium with $0.8 \%$ agarose, devoid of plant hormone supplements.

2. Sterilize the $D$. caryophyllus seeds using three-step procedure: a 1 min wash in $70 \%$ ethanol, followed by a 4 min wash in $20 \% \mathrm{NaClO}$, and a final rinse in sterile distilled water 3 times.

3. Allow the $D$. caryophyllus seeds to germinate on the MS media by incubating the plates at $25^{\circ} \mathrm{C}$ in $14 \mathrm{~h}$ light and $10 \mathrm{~h}$ dark cycles in a plant culture room, until the root length reaches $2 \mathrm{~cm}$ (approximately six days).

4. Treat the germinated plant roots by immersing the root tips into $5 \mathrm{ml}$ of the bacterial suspension with titre $10^{5} \mathrm{CFU} \mathrm{ml}^{-1}$ in the universal tube.

5. Transfer the treated plants onto new MS agar medium devoid of IAA and sucrose in square Petri plates (120 mm x $120 \mathrm{~mm} \times 15 \mathrm{~mm}$ ).

6. Again, incubate the plates at $25^{\circ} \mathrm{C}$ in $14 \mathrm{~h}$ light and $10 \mathrm{~h}$ dark cycles in a plant culture room.

7. After two weeks of infection, stain the treated roots with Salkowski reagent.

8. To stain the roots, add $400 \mu \mathrm{l}$ of Salkowski reagent on each root and observe any visible color change.

9. The development of pink color as shown in the figure below indicates the presence of proximal IAA on and around the roots (Figure 2).

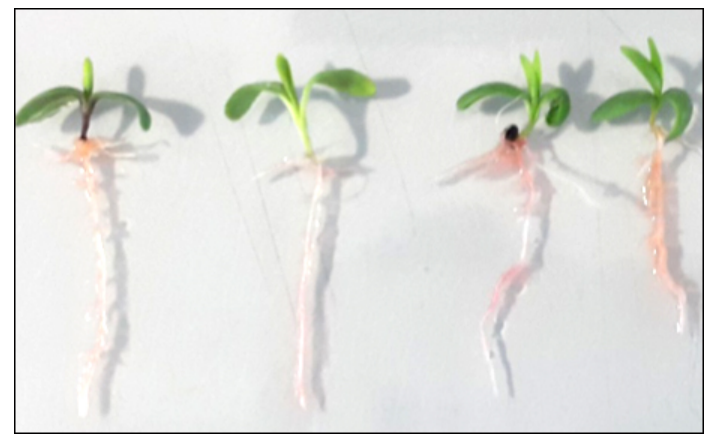

Figure 2. In situ Salkowski staining on plant roots treated with Klebsiella SGM 81 of

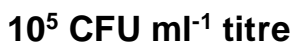

\section{Data analysis}

1. Take the optical density of the samples at $536 \mathrm{~nm}$ and graphically calculate the concentration of IAA based on the standard curve. IAA estimation from Klebsiella SGM 81 strain, using 
Salkowski reagent shows a maximum yield to $215 \mu \mathrm{g} \cdot \mathrm{ml}^{-1}$ detected after $72 \mathrm{~h}$ with $0.15 \%$ tryptophan (Figure 1a in Gang et al., 2018).

2. For LC-MS/MS analysis, confirmation of the identity in the samples is done by Enhanced Product Ion scans.

3. Data acquisition and analysis is done with Analyst 1.6.1 (AB Sciex).

4. Quantification is carried out by comparing the test sample data with control standard IAA.

5. Overlapping retention times during LC (5.90 $\mathrm{min}$ and $5.92 \mathrm{~min}$ ) and identical $\mathrm{m} / \mathrm{z}$ ratios (130.1) between a commercial IAA standard and sample supernatant showed Klebsiella SGM 81 to produce substantial amounts (960 $\mu \mathrm{g} \cdot \mathrm{ml}^{-1}$ after $72 \mathrm{~h}$ ) homogeneous IAA (Gang et al., 2018).

6. Development of pink color on the Salkowski treated roots indicates the presence of bacterial IAA proximal to the roots (Figure $3 a$ in Gang et al., 2018).

\section{$\underline{\text { Notes }}$}

1. The Salkowski reagent method discussed in this paper is shown using nutrient broth liquid medium (supplemented with L-tryptophan) which is non-specific liquid medium for most grampositive and gram-negative bacteria. However other media like Yeast extract mannitol (YEM), Pikovskaya's broth (supplemented with L-tryptophan) can be used if IAA production and quantification is to be done from Rhizobium or phosphate solubilizing bacteria respectively.

2. The preculture preparation is important to normalize the initial cell count especially when a comparative study of IAA quantification is carried out for the optimization process.

3. Salkowski reagent method quantifies the overall Indole present in the sample. To get the purity and conjugants of Indole-3-acetic acid (IAA), other analytical methods like HPLC, LC-MS/MS, HPTLC etc. can be used.

\section{Recipes}

1. Culture media

a. Making use of measuring cylinder, measure $100 \mathrm{ml}$ distilled water and transfer it into a $250 \mathrm{ml}$ Erlenmeyer flask or screw-capped glass bottle. Two other flasks of the same media, with and without tryptophan will be used as control and pre-culture media (used to activate the SGM 81 culture to yield young culture) respectively

b. For simple liquid nutrient broth medium, weigh $1.3 \mathrm{~g}$ of Nutrient broth and dissolve it in $100 \mathrm{ml}$ distilled water. Stir it properly so as to mix the powder in distilled water properly

c. Weigh $0.15 \mathrm{~g}$ of L-tryptophan $(0.15 \% \mathrm{w} / \mathrm{v})$ and add to the prepared nutrient broth flask. This is liquid nutrient broth with tryptophan supplement

d. Autoclave the prepared media at $121^{\circ} \mathrm{C}$ for $15 \mathrm{~min}$

e. Allow the media to cool down and come to room temperature before inoculation so as to ensure the survival of inoculum 
2. Salkowski reagent

a. $\quad 0.5 \mathrm{M}$ of $100 \mathrm{ml}$ ferric chloride (dissolve $8.125 \mathrm{~g}$ of $\mathrm{FeCl}_{3}$ in $100 \mathrm{ml}$ of distilled water)

b. To dilute readily available perchloric acid, measure $24.5 \mathrm{ml}(\mathrm{v} / \mathrm{v})$ of distilled water in a measuring cylinder and add $24.5 \mathrm{ml}(\mathrm{v} / \mathrm{v})$ of concentrated acid to it

c. Add $1 \mathrm{ml}(\mathrm{v} / \mathrm{v})$ of $0.5 \mathrm{M}$ ferric chloride solution to $49 \mathrm{ml}$ of $35 \%$ perchloric

d. Mix well and store in a dark brown bottle at room temperature. It can be used for further experiments if stored under described conditions

\section{Acknowledgments}

We are thankful to the Department of Science and Technology, India for the financial assistance under INSPIRE Fellowship 2014-15 (IF 140042). This work was also supported by the BBSRC (BB/003608/1), UK. At Imperial College London, we thank Mark Bennett for helping with LCMS/MS analysis. The protocol is adapted from Gang et al. (2018).

\section{Competing interests}

We declare no conflict of interest.

\section{References}

1. Chiwocha, S. D., Abrams, S. R., Ambrose, S. J., Cutler, A. J., Loewen, M., Ross, A. R. and Kermode, A. R. (2003). A method for profiling classes of plant hormones and their metabolites using liquid chromatography-electrospray ionization tandem mass spectrometry: an analysis of hormone regulation of thermodormancy of lettuce (Lactuca sativa L.) seeds. Plant J 35(3): 405417.

2. Gang, S., Saraf, M., Waite, C. J., Buck, M. and Schumacher, J. (2018). Mutualism between Klebsiella SGM 81 and Dianthus caryophyllus in modulating root plasticity and rhizospheric bacterial density. Plant Soil 424: 273-288.

3. Gordon, S. A. and Weber, R. P. (1951). Colorimetric estimation of indoleacetic acid. Plant Physiol 26(1): 192-195.

4. Goswami, D., Thakker, J. N. and Dhandhukia, P. C. (2015). Simultaneous detection and quantification of indole-3-acetic acid (IAA) and indole-3-butyric acid (IBA) produced by rhizobacteria from I-tryptophan (Trp) using HPTLC. J Microbiol Methods 110: 7-14.

5. Kallenbach, M., Baldwin, I. T. and Bonaventure, G. (2009). A rapid and sensitive method for the simultaneous analysis of aliphatic and polar molecules containing free carboxyl groups in plant extracts by LC-MS/MS. Plant Methods 5: 17.

6. Kamilova, F., Kravchenko, L. V., Shaposhnikov, A. I., Azarova, T., Makarova, N. and Lugtenberg, B. (2006). Organic acids, sugars, and L-tryptophane in exudates of vegetables growing on 
stonewool and their effects on activities of rhizosphere bacteria. Mol Plant Microbe Interact 19(3): 250-256.

7. Kamnev, A., Shchelochkov, A., Perfiliev, Y. D. Tarantilis, P. A., and Polissiou, M. G. (2001). Spectroscopic investigation of indole-3-acetic acid interaction with iron (III). J Mol Struct 563:565-572.

8. Kravchenko, L., Azarova, T., Makarova, N. and Tikhonovich, I. (2004). The effect of tryptophan present in plant root exudates on the phytostimulating activity of rhizobacteria. Microbiology 73:156-158.

9. Lynch, J. (1985). Origin, nature and biological activity of aliphatic substances and growth hormones found in soil. In: Soil Organic Matter and Biological Activity. Springer 151-174.

10. Perrig, D., Boiero, M. L., Masciarelli, O. A., Penna, C., Ruiz, O. A., Cassan, F. D. and Luna, M. V. (2007). Plant-growth-promoting compounds produced by two agronomically important strains of Azospirillum brasilense, and implications for inoculant formulation. Appl Microbiol Biotechnol 75(5): 1143-1150.

11. Spaepen, S., Vanderleyden, J. and Remans, R. (2007). Indole-3-acetic acid in microbial and microorganism-plant signaling. FEMS Microbiol Rev 31(4): 425-448. 